\section{COMPETENCIA POLÍTICA Y PRESUPUESTO DE VIVIENDA EN AÑOS ELECTORALES EN CHILE ENTRE 1990 Y 2015}

Juan Pablo Urrutia ${ }^{1}$

\section{Resumen}

Los presupuestos nacionales para vivienda responden a muchas variables además del propio déficit habitacional. La competencia electoral es una de ellas, por lo cual su estudio toma relevancia para comprender uno de los orígenes de la inversión en vivienda. Para ello, se escoge el caso de Chile, que mediante un estudio cuantitativo-descriptivo con análisis correlacional utiliza información secundaria sobre su presupuesto público desde 1990 al año 2015 y los resultados de elecciones presidenciales de aquel periodo. Los resultados sugieren que los gobiernos manejaron la glosa de vivienda del presupuesto nacional en periodos electorales, acción

\section{POLITICAL COMPETITION AND HOUSING BUDGET DURING ELECTION YEARS IN CHILE FROM 1990 TO 2015}

Juan Pablo Urrutia ${ }^{1}$

\section{Abstract}

The national housing budget is determined by many variables in addition to that of housing deficit. Electoral competition is one of these variables and thus becomes a key aspect to study in order to understand one of the driving forces behind housing investment. Through quantitative-descriptive methods and correlational analysis, this paper uses secondary information to examine the Chilean public budget and the results of presidential elections over the 1990-2015 period. The results suggest that each administration modified the housing budget during electoral periods, a situation that grew in intensity when there was 
acentuada cuando la oposición fue más fuerte. Además, es posible observar que el oficialismo envió "señales costosas" con grandes inversiones en subsidios habitacionales para dar constancia de sus capacidades, pero al mismo tiempo, tal como en un "juego repetitivo", los votantes observaron que aquellos esfuerzos ocurrían solo en años electorales, por lo que las señales podrían haber sido interpretadas como populismo electoral en vez de una demostración de eficacia.

PALABRAS CLAVE: PRESUPUESTO; VIVIENDA; SIGNALING; PANDERING; ELECCIONES

Recibido: 04-09-2018

Aceptado: 17-10-2018

1 Chile. Profesor asistente, Facultad de Arquitectura y Urbanismo Universidad de Chile. ORCID https://orcid.org/0000-0003-2019006X. Correo electrónico: jpurrutia@uchile.cl. greater opposition. In addition, it is possible to observe that the ruling parties demonstrated their economic power through "costly gestures" with major investments and the provision of housing subsidies to validate their capacity. However at the same time, like in a "repetitive game" voters observed that these efforts were only made during electoral years, thus being interpreted as electoral populism instead of a demonstration of efficacy.

KEYWORDS: BUDGET; HOUSING; SIGNALING; PANDERING; ELECTION

Received: 04-09-2018

Accepted: 17-10-2018

1 Chile. Assistant Professor, Faculty of Architecture and Urban Planning, University of Chile. ORCID https://orcid.org/00000003-2019-006X. Email: jpurrutia@uchile.cl. 


\section{Importancia de la relación entre elecciones e inversión en vivienda}

Existen muchos estudios acerca de los ciclos presupuestarios políticos desde Nordhaus (1975). La mayoría de ellos describe y explica por qué y cómo los gobiernos manejan el presupuesto público en años electorales con el fin de incrementar la probabilidad de reelección. Sin embargo, cabe preguntarse si acaso los gobiernos manejan el presupuesto público para enviar una señal de eficacia o es simplemente populismo con fines electorales. Estas preguntas podrían ser respondidas identificando un caso real para analizarlo a la luz de la teoría del signaling, que estudia las acciones políticas como señales de eficiencia, y el pandering, que analiza las decisiones populistas adoptadas por autoridades como acciones exclusivas para complacer la opinión pública por sobre la búsqueda del bien común.

Por lo tanto, resulta interesante estudiar el comportamiento de las glosas presupuestarias de vivienda en Chile durante años electorales, su relación con el déficit habitacional y la competitividad política. Para ello el caso de Chile es ilustrativo dado sus reconocidos logros en materia de política habitacional, además de ser una nación que se enfrentó al aprendizaje de vivir en democracia después de una dictadura, periodo democrático que comenzó con dos décadas gobernadas por partidos opositores al régimen autoritario, que luego fueron sucedidos por un gobierno de partidos ligados a la pasada dictadura, y este a su vez reemplazado por una administración de la oposición. Es decir, después de 20 años se transitó a un periodo de alternancia en el poder, mientras que el déficit de vivienda en el país fue decayendo en consecuencia con los niveles de desarrollo alcanzado.

En el contexto chileno de gobiernos democráticos entre 1990 y 2015, se intenta comprender cómo el manejo presupuestario, especialmente en vivienda, podría responder a la entrega de una señal de eficiencia o a un acto populista que no responde a una distribución necesariamente responsable de recursos. Esta diferencia depende del momento en que la acción presupuestaria es realizada, la cantidad de periodos de la acción con los mismos actores, la fortaleza de la coalición opositora que representaría una amenaza para el oficialismo y la relación entre las preferencias de los votantes y la información que posee el gobierno. Solo escasos estudios han incluido el grado de competitividad electoral como un factor que afecta el manejo del presupuesto público (Aidt, Veiga, y Veiga, 2011; Eibl y Lynge-Mangueira, 2017) y la idea de que la manipulación del presupuesto no es solo relevante en su glosa total, sino que también es acerca de la distribución del gasto entre sectores específicos (Vergne, 2009; Katsimi y Sarantides, 2012; Castro 
y Martins, 2016). Para el caso específico chileno no se han realizado estudios de este tipo que vinculen al presupuesto de vivienda, sino que solo considerado el presupuesto a nivel global en estudios de panel en comparación a otros países en desarrollo o del grupo latinoamericano.

\section{Metodología}

La investigación que respalda este trabajo intenta comprender un fenómeno político social desde una aproximación cuantitativa con un estudio descriptivo, para lo cual se utilizan datos secundarios de fuentes oficiales del gobierno, con el objeto de describir la relación entre competitividad política y presupuesto habitacional mediante análisis correlacional de resultados electorales presidenciales y de inversión en vivienda para dichos periodos. La indagación sobre el fenómeno de la distribución del presupuesto y sus razones se basa en las siguientes dos preguntas que detonan este estudio: ¿Cómo los gobiernos chilenos han manejado el presupuesto público para el desarrollo habitacional en años electorales?, ¿Esto lo hacen con el fin de enviar una señal de eficiencia o es un acto populista? Si bien estas interrogantes no tienen el propósito de explicar la causalidad del fenómeno, sus respuestas pueden ayudar a comprender la relación entre elecciones e inversión en vivienda. Para ello se realiza un análisis correlacional entre la evolución del crecimiento del producto interno bruto de Chile, glosas del presupuesto público nacional, inversión en programas de vivienda del gobierno y la evolución del déficit habitacional en el país, todos con un procesamiento a nivel de series anuales comparativas en la línea temporal entre los años 1990 y 2015, datos secundarios que además son complementados con los resultados electorales de las seis elecciones presidenciales del periodo. Dicho análisis se desarrolla a la luz de lo que plantea el estado del arte en el estudio sobre los ciclos de presupuesto público (CPP, en inglés Public Budget Cycles o PBCs) y las teorías del signaling y el pandering en política para entender las diferencias e implicancias de estos dos modelos aplicados al ámbito de política habitacional. De esta forma, se busca comprender la lógica del manejo presupuestario e identificar las eventuales motivaciones políticas detrás de la asignación de recursos para la vivienda.

\section{Signaling y pandering en el presupuesto público}

Los ciclos de presupuesto público (CPP) han sido estudiados durante las últimas cuatro décadas. En un comienzo fueron definidos como los efectos de las elecciones en la economía a través de la manipulación de variables macroeconómicas por el 
oficialismo con el fin de tomar ventaja para las elecciones (Nordaus, 1975). Luego a fines de los años ochenta, Rogoff (1987) y Rogoff y Sibert (1988) indicaron que los gastos públicos pre-electorales son una señal de la eficiencia de un gobierno para distribuir bienes y servicios públicos. Otros autores, tales como Persson y Tabellini (1990), sugieren lo mismo, pero incluyendo la idea de que los juegos repetidos involucran a la reputación como una variable, por lo tanto, se reduce el efecto de la señal. Estos autores demostraron que los CPP son afectados por las normativas electorales y los tipos de gobiernos, de esta forma, los regímenes presidenciales y sistemas proporcionales aumentan la probabilidad del manejo presupuestario para incrementar el gasto público en años electorales (Persson y Tabellini, 2003). Otra característica descubierta fue que los CPP ocurren con mayor frecuencia en las nuevas democracias, esto significa que aquellos países están aprendiendo a ser democráticos después de estar largo tiempo bajo regímenes autoritarios (Brender y Drazen, 2005; Shi y Svensson 2006). Finalmente, en estudios de Shi y Svensson (2002 y 2006) y Vergne (2009), se sugiere que en los países en desarrollo el manejo del presupuesto público es más alto.

Un importante cambio en la investigación de los CPP comenzó en la década pasada con la distinción entre el manejo del presupuesto total y la asignación de esos recursos según cartera. Por ejemplo, Khemani (2004) descubrió que en India las autoridades políticas locales manejan el gasto público en años electorales con el fin de utilizar políticas públicas específicas dirigidas a satisfacer grupos de interés particulares sin que ello implique un aumento en el presupuesto total de su administración. En ese estudio se demostró que los departamentos de obras públicas utilizan más recursos para invertir en la construcción de caminos en momentos pre-electorales. También Vergne (2009) manifiesta que las elecciones tienen un impacto en la asignación del gasto público, pero no necesariamente en el presupuesto completo, lo que significa que las autoridades desplazan recursos desde las inversiones de infraestructura hacia gastos en salarios y subsidios dado que estos son más visibles a corto plazo para el electorado. En la misma línea, Katsimi y Sarantides (2012), y recientemente Castro y Martins (2016), indican cómo las autoridades manejan el presupuesto para años electorales derivando recursos hacia bienes más visibles mientras que el gasto en inversiones de capital se disminuye dado que estas requieren de más tiempo para materializarse.

En contraste, Drazen y Eslava (2010) demuestran que las elecciones afectan la distribución del gasto público incrementando recursos para grandes inversiones en construcción en vez de gastos relacionados a la vida cotidiana de las personas como los salarios y subsidios. En este caso los autores muestran que algunas partidas del presupuesto aumentan en años electorales como salud en $7 \%$, 
infraestructura de aguas y energía en 5\% y vivienda en casi 20\%, mientras que algunas partidas relacionadas a gastos de corto plazo y directos a las personas disminuyen sus recursos. Finalmente, un interesante enfoque sobre CPP tiene que ver con la relación entre el nivel de competitividad y la magnitud de la manipulación del presupuesto público. Diversos estudios (Aidt et al., 2011; Efthyvoulou, 2012; Eibl y Lynge-Mangueira, 2017) prueban que las autoridades tienen comportamientos más oportunistas en años electorales cuando la confianza en el triunfo es menor y el resultado posterior tiene un pequeño margen dado una elección reñida.

De acuerdo con la principal pregunta de esta investigación, si el manejo del presupuesto es una señal para demostrar eficiencia o simplemente populismo, es necesario entender la naturaleza y teorías de cada uno de estos enfoques en profundidad. Primero, cabe recordar que el signaling o juego de señales fue desarrollado en 1973 por el Premio Nobel de Economía Michael Spence. La idea general trata sobre la asimetría de información entre los jugadores, desalineación de sus intereses y la oportunidad de enviar una señal (Spence, 1973). Para explicar esto en términos de los CPP los jugadores deberían constituirse por un lado con la autoridad (persona, partido o coalición) y por el otro el electorado quienes no poseen la misma información acerca del contexto político y desempeño de la autoridad. La autoridad desea permanecer en el poder a pesar de la calidad de su desempeño, por lo tanto los jugadores no comparten los mismos intereses, ya que el electorado preferiría escoger a los gobernantes de acuerdo a su desempeño, castigando en las urnas a la coalición que no haya hecho bien su trabajo. Por último, la autoridad tiene la posibilidad de enviar una señal a través del presupuesto público para un año electoral. Según esto es posible inferir que el manejo del presupuesto podría ser analizado como un instrumento de señalización tal cual lo indican diversos autores (Rogoff, 1987; Rogoff y Sibert, 1988; Persson y Tabellini, 2003; Shi y Svensson 2006).

Luego, el modelo del pandering o juego del populismo es explicado por Canes-Wrone, Herron y Shotts (2001) y Maskin y Tirole (2004). De ambos trabajos es posible inferir que las condiciones para distinguir la presencia de populismo se manifiestan cuando hay asimetría de información entre los votantes y la autoridad, la posibilidad de este último de tomar acciones unilaterales, existencia de incentivos electorales, y la posibilidad de selección negativa por la autoridad, en otras palabras, posibilidad de inconsistencia entre las creencias de los políticos y las preferencias de los votantes. Por lo tanto, el manejo del gasto público podría ser considerado como un acto populista solo si la autoridad se comporta de manera oportunista asignando recursos influenciada solo por las preferencias de la gente en vez de la información que maneja como experto, la que no representa necesariamente el deseo espontaneo popular. 
Tomando en consideración ambas posibilidades de interpretación sobre las acciones para definir el presupuesto en años electorales, es posible afirmar que la cantidad de periodos analizados es el elemento clave para diferenciar entre la teoría de las señales y del populismo. Si se analiza solo un periodo, lo más probable es que el manejo del presupuesto sea entendido nada más que como una señal de eficiencia, pero cuando aumenta la cantidad de periodos observados entra al juego la variable reputación, donde los votantes disponen del tiempo suficiente para observar el comportamiento una y otra vez del gobernante y cómo este es consecuente o no entre sus acciones y creencias. Por lo tanto, es posible afirmar que pasar de un juego estático (un solo periodo) a otro dinámico (dos o más periodos) -donde el electorado tiene la posibilidad de aprender-implica el aumento de la probabilidad de identificar la presencia de populismo.

Una sociedad que ya ha transitado por una serie de gobiernos democráticos ha estado aprendiendo sobre la democracia y madurando junto a las instituciones que garantizan todas sus condiciones, de esa forma los niveles de democratización aumentan (Brender y Drazen, 2005; Alt y Lassen, 2006), por lo cual la ciudadanía adquiere progresivamente una cultura cívica que le permite tener mayor consciencia y capacidad de identificar y cuestionar el comportamiento de sus gobernantes (Persson y Tabellini, 1990; Alt y Lassen, 2006). Por lo tanto, un electorado maduro en términos democráticos, sería capaz de distinguir entre una señal de eficiencia y un acto de populismo. Por ejemplo, si el electorado tuviese la oportunidad de observar en más de una ocasión el comportamiento de un gobierno durante años electorales, podría llegar a ser capaz de identificar actos de manejo del presupuesto o incluso -si el nivel de acceso a la información lo permite- de diferenciar entre inversiones en beneficios tangibles a corto plazo y asignación de recursos para las glosas más necesarias para la población, lo que por consecuencia permitiría la distinción entre señales de eficiencia y actos populistas en años electorales.

\section{El caso chileno}

Como es internacionalmente conocido, Chile estuvo regido bajo una dictadura durante 17 años desde 1973, cuando las Fuerzas Armadas dieron un golpe de Estado al gobierno de Salvador Allende. En ese periodo, entre los años 1973 y 1989, hubo espacio solo para tres votaciones populares, la primera para apoyar o no al régimen en 1978, la segunda para validar la nueva constitución en 1980, y la tercera para consultar sobre la continuidad en el gobierno de Augusto Pinochet en 1988. El resultado de este último referéndum rechazó a la dictadura, por lo que en 1989 comenzó la transición hacia una nación democrática a través de elecciones para un nuevo gobierno y parlamento. 
Justo antes de la elección presidencial tuvo lugar un nuevo referéndum, que aprobó reformas constitucionales que establecieron el principio de democracia y un nuevo sistema electoral para Chile.

El sistema presidencial fue el modelo democrático escogido, el que incluyó tres niveles de elecciones: 1) a nivel local, para elegir a las autoridades municipales; 2) a nivel distrital, para escoger a los representantes en el parlamento; y 3) a nivel nacional, para votar por el presidente. En el primer estrato los alcaldes son electos por mayoría simple, a diferencia del tercer nivel, donde el presidente debe obtener la mayoría absoluta para ser electo. El sistema electoral parlamentario requiere de mayor explicación dada su complejidad e impacto en la configuración de los actores de la arena política chilena. Primero, se debe entender que las cámaras se definieron con dos puestos por cada distrito electoral donde los votantes deben volcar sus preferencias por un solo candidato que a su vez pertenece a una lista. Los dos candidatos más votados de la lista con mayoría de sufragios serán electos como diputados o senadores si es que su lista dobla en votos a la lista que la secunde. De no ser así se elige al candidato con más sufragios individuales de cada una de las dos listas con más votos.

Este modelo electoral para definir los puestos en el parlamento genera dos importantes consecuencias que afectaron la configuración del escenario político chileno en cuanto a la definición de actores y sus incentivos. La primera consecuencia es la generación de dos grandes y poderosas coaliciones dada la necesidad de asociarse en listas para obtener los dos escaños por distrito electoral. Entre ambas obtuvieron prácticamente todos los puestos del Congreso desde 1990, periodo en el cual la agenda legislativa dependió exclusivamente de estas dos coaliciones políticas. Ambos grupos corresponden a la Concertación de Partidos por la Democracia (Concertación) y la Alianza por Chile 2 (Alianza), quienes gozaron de una inmensa hegemonía política en el país, sumado a una gran cohesión interna especialmente en tiempos de campaña electoral mientras duró ese sistema que fue reemplazado en 2017, por lo que y a pesar de ser coaliciones de partidos con diferencias entre ellos, los grupos se comportaron como si cada uno fuese un solo partido.

2 La Alianza por Chile tomó diversos nombres entre 1990 y 2015 a pesar de estar compuesta siempre por los mismos partidos (Unión Demócrata Independiente y Renovación Nacional), salvo algunas excepciones. Por ello, esta coalición puede encontrarse como "Democracia y Progreso", "Participación y Progreso", “Unión por el Progreso de Chile”, “Unión por Chile”, “Coalición por el Cambio" y, actualmente, como "Chile Vamos". 
La segunda consecuencia dice relación con la clara configuración de una gran coalición de oposición, la que operó a modo de fuerzas alineadas para estar constantemente fiscalizando la labor del oficialismo, algo muy distante a lo que sucede en países con sistemas electorales proporcionales, donde la oposición tiende a conformarse por una suma de diversos partidos desarticulados entre ellos. Así es como el contexto político chileno se configuró prácticamente con solo dos actores a contar de 1990, donde la Concertación gobernó durante 20 años con una clara oposición conformada por los partidos de derecha, situación que persistió hasta el año 2010, cuando Sebastián Piñera -líder de la Alianza- fue electo presidente, quien a su vez fue sucedido por Michelle Bachelet dando paso a una fase de alta alternancia en el periodo analizado.

Los presidentes en Chile son elegidos por mayoría absoluta. Si esta no se cumple en una primera ronda de votaciones existe la posibilidad de una segunda vuelta de sufragios con las dos primeras mayorías. Los presidentes no pueden ser reelectos para el siguiente periodo, sin embargo, un ex presidente podría volver a competir en el periodo subsiguiente. Esta situación de alternancia obligada no afecta el comportamiento electoral de los presidentes debido a que, como el contexto político está compuesto principalmente por dos coaliciones fuertes y cohesionadas, el presidente siempre vuelca todo su apoyo al candidato de su coalición con el fin de asegurar la permanencia en el gobierno de su grupo de partidos. En un comienzo, justo después del régimen de Pinochet, el primer periodo de transición presidencial duró cuatro años, luego los siguientes periodos fueron de seis años, pero en 2005 nuevamente volvieron a regirse por periodos de cuatro años con el fin de aumentar la productividad de los gobiernos. Así, han pasado seis elecciones desde 1989 hasta 2015, con los resultados que muestra la Tabla 1.

Cada uno de los gobiernos tiene el poder de definir el presupuesto público en $\mathrm{Chile}^{3}$ que es diseñado cada año por el poder ejecutivo a través de la Dirección de Presupuesto del Ministerio de Hacienda (Chile Ministerio de Hacienda, s.f.). Luego, el plan de gasto se presenta al parlamento para su aprobación, donde los diputados y senadores solo podrían hacer refrendas para disminuir el presupuesto, sin el poder para aumentarlo o eliminar partidas. La decisión es tomada por mayoría absoluta y si no hay acuerdo dentro de los plazos establecidos se implementa el primer proyecto que el ejecutivo

3 Esta información está en valores reales al 2015 (PIB y presupuesto), por lo tanto sus montos no están distorsionados por la inflación. 
TABLA 1: RESULTADOS DE LAS ELECCIONES PRESIDENCIALES Y MARGEN DE VICTORIA

\begin{tabular}{|c|c|c|c|c|c|c|}
\hline & 1989 & 1993 & $1999-2000$ & $2005-2006$ & $2009-2010$ & 2013 \\
\hline \multirow{3}{*}{$\begin{array}{l}\text { Resultado } \\
\text { final }\end{array}$} & Aylwin 55,17\% & Frei $57,98 \%$ & Lagos $51,31 \%$ & Bachelet 53,50\% & Piñera 51,61\% & Bachelet $62,17 \%$ \\
\hline & Büchi 29,40\% & Alessandri $24,41 \%$ & Lavín 48,69\% & Piñera 46,50\% & Frei 48,39\% & Matthei 37,83\% \\
\hline & Errázuriz 15,43\% & Otros (4) $17,60 \%$ & & & & \\
\hline $\begin{array}{l}\text { Margen de } \\
\text { victoria }\end{array}$ & 25,77 puntos & 33,57 puntos & 2,62 puntos & 7,00 puntos & 3,22 puntos & 24,34 puntos \\
\hline $\begin{array}{l}\text { Coalición } \\
\text { política }\end{array}$ & $\begin{array}{l}\text { Concertación } \\
\text { (centroizquierda) }\end{array}$ & $\begin{array}{l}\text { Concertación } \\
\text { (centroizquierda) }\end{array}$ & $\begin{array}{l}\text { Concertación } \\
\text { (centroizquierda) }\end{array}$ & $\begin{array}{l}\text { Concertación } \\
\text { (centroizquierda) }\end{array}$ & $\begin{array}{l}\text { Alianza por Chile } \\
\text { (centroderecha) }\end{array}$ & $\begin{array}{l}\text { Concertación } \\
\text { (centroizquierda) }\end{array}$ \\
\hline
\end{tabular}

Fuente: Elaboración propia a partir de datos del Servicio Electoral de Chile.

elaboró. Por lo tanto, la autoridad prácticamente tiene el poder absoluto para controlar el presupuesto público de cada año.

En materia de desarrollo el país comenzó su etapa democrática con altos índices de pobreza y un Producto Interno Bruto (PIB) per cápita aproximado de 4.000 USD (a precio constante del 2005), elementos que sin duda contribuyeron a la agenda programática de los gobiernos de la Concertación, los que se caracterizaron por mantener un crecimiento sostenido especialmente durante los noventa, lo que permitió aumentar el PIB per cápita de 4.062 USD al inicio de los noventa a 8.677 USD en el año 2010. Al mismo tiempo los indicadores de pobreza se redujeron pasando de 38,6\% de personas pobres en 1990 a 14,4\% veinte años más tarde según datos del Ministerio de Desarrollo Social. Todo indica que el país fue progresando y sus indicadores de desarrollo permiten argumentar que los problemas de pobreza más graves fueron atendidos.

Uno de los grandes problemas sociales heredados del periodo de Pinochet fue el gran déficit habitacional, el que alcanzó un nivel de requerimientos al borde del millón de unidades habitacionales, déficit que estaba compuesto principalmente por hogares y núcleos familiares que vivían en allegamiento. Por tanto, la instauración de un sistema democrático en el país supuso un riesgo inminente de tomas informales de terreno para aliviar aquella 
inmensa demanda social contenida por un periodo autoritario. Por ello, en los diez primeros años de gobiernos de la Concertación se diseñaron políticas habitacionales orientadas principalmente a la masificación de viviendas con producción de corto plazo (Chile Ministerio de Vivienda y Urbanismo [MINVU], 2004). En tanto en el segundo gobierno de la Concertación la meta propuesta fue de 90.000 soluciones habitacionales por año (Chile Ministerio de Planificación y Cooperación [MIDEPLAN], 1998) y que se logró alcanzar gracias al crecimiento económico vigoroso del país en la década del noventa, dejando a Chile como el primer país latinoamericano en disminuir el déficit habitacional. Como resultado de ese periodo se obtuvo una enorme cantidad de viviendas en muy poco tiempo, especialmente para el $40 \%$ más pobre de la población. Con un país económicamente ejemplar a nivel latinoamericano, pero vergonzoso mundialmente en niveles de inequidad, los gobiernos posteriores reorientaron sus esfuerzos en otorgar calidad e integración al desarrollo de vivienda social, ya que hasta entonces prácticamente todos los esfuerzos estuvieron en torno solo a la cantidad de viviendas (MINVU, 2004).

En resumen, el contexto político en Chile en el periodo estudiado se compone de solo dos actores en forma de coaliciones, donde una juega el rol del oficialismo y la otra de una oposición organizada. Quien está en el gobierno tiene el poder de definir el presupuesto y su composición, mientras que la oposición representa una amenaza en cada contienda electoral, en un escenario con un Chile cada vez más desarrollado y maduro en términos democráticos.

\section{Análisis de presupuestos en años electorales en Chile}

Mientras el PIB ${ }^{4}$ de Chile fue creciendo alrededor del $8 \%$ anual en los años noventa, el presupuesto también se incrementó pero a un ritmo menor. Sin embargo, al observar las series anuales, en 1993 el gasto público aumentó en una mayor proporción que el del PIB, al igual que en 1999, año en que se realizó la tercera elección presidencial, donde el presupuesto se incrementó en casi un 7\% respecto del año anterior mientras que el crecimiento del PIB fue negativo. Posteriormente, en la década del 2000, el crecimiento del PIB y presupuesto tuvieron ritmos más bajos que en la década pasada. A pesar de ello el comportamiento fue similar, en

4 Para comparar el crecimiento del PIB con el del presupuesto, no se consideraron los impuestos como una variable, debido a que en Chile no hubo reformas tributarias importantes en el periodo analizado. 
años electorales el crecimiento anual del gasto público fue mayor al del PIB como sucedió en el año 2005, en efecto, en 2009 el gasto público se elevó casi en un $17 \%$, mientras que el PIB tuvo crecimiento negativo, muy por el contrario a lo ocurrido en 2013, cuando ambos indicadores crecieron en la misma magnitud (Figura 1).

Por lo tanto, podría ser posible sugerir que el presupuesto global fue manejado por las autoridades con el fin de complacer a los votantes a excepción del año 2013. Los antecedentes que contribuyen a esta aseveración corresponden a que en las cuatro primeras elecciones presidenciales (1993, 1999, 2005 y 2009) el PIB presentó un crecimiento inferior al del año anterior, pero a pesar de ello los gobiernos decidieron incrementar el presupuesto con un nivel de crecimiento superior al del año precedente.

Más allá del comportamiento del presupuesto global respecto de la variación del crecimiento del PIB, hay una arista interesante de analizar acerca de la composición del presupuesto tal como sugieren diversos estudios (Khemani, 2004; Vergne, 2009; Drazen y Eslava, 2010; Katsimi y Sarantides, 2012; Castro y Martins, 2016), que afirman que las autoridades no solo controlan el presupuesto total, sino que también manejan sus componentes reorientando recursos desde algunas partidas a otras con mayor potencial para capturar votantes. En Chile es posible observar cómo algunos ítems del presupuesto tienen un singular comportamiento en años electorales, por ejemplo las partidas presupuestarias de asistencia social siguen la tendencia del presupuesto global en años electorales, es decir que aumentan con él y, más aún, sus incrementos son más dramáticos que los del presupuesto total, mientras que partidas como la de educación suelen mantenerse al mismo nivel de gasto que años anteriores o incluso disminuyen su crecimiento presupuestario antes de un periodo electoral (Figura 2). Este comportamiento de las glosas sugiere la idea de que las autoridades no solo aumentan los recursos en algunas partidas, sino que también reducen el crecimiento para ciertos sectores en años electorales.

Dada la estrategia electoral de manejar el presupuesto asignando con más atención a partidas específicas a costa de otras, justo antes de las elecciones, se infiere que el oficialismo distribuiría los recursos públicos con el fin de favorecer un mayor impacto en los votantes. Tal como se presenta en la Figura 2, en años electorales el presupuesto orientado a la asistencia social tiene mayor crecimiento que el gasto en educación a excepción de lo ocurrido en el año electoral 2013. En este sentido las autoridades estuvieron más preocupadas por los resultados a corto plazo que por los resultados observables en un plazo mayor al de sus mandatos. Por esta razón, en el estudio se seleccionó un sub-ítem del presupuesto de asistencia social que puede mostrar cuán atentas estaban las autoridades sobre las elecciones y las probabilidades que tenían de ganar para continuar en el poder. 


\section{FIGURA 1. CRECIMIENTO ANUAL DEL PIB Y PRESUPUESTO PÚBLICO EN CHILE.}

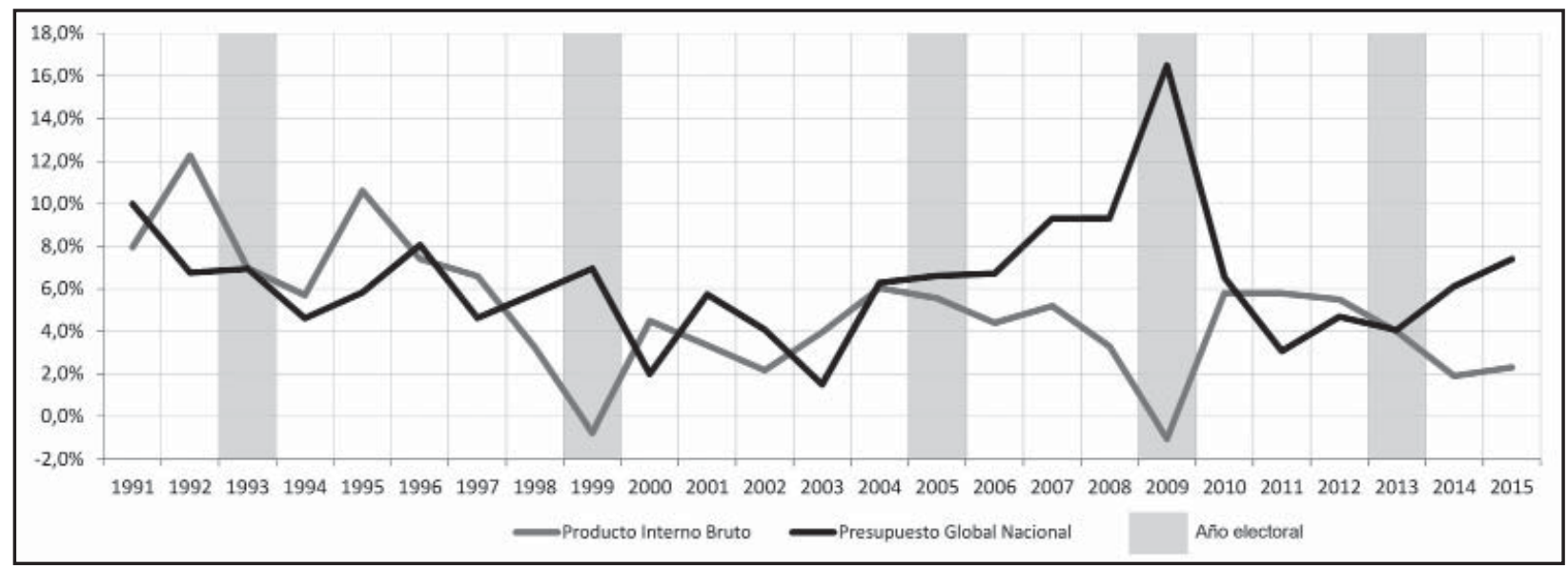

Fuente: Elaboración propia en base a datos del Banco Mundial (s.f.) y Chile Ministerio de Hacienda, s.f.

La partida específica dentro del presupuesto de asistencia social que refleja aquel comportamiento electoral es la glosa sobre vivienda, en este caso, el crecimiento del gasto público a través de subsidios y construcción fue relativamente similar en las dos primeras elecciones presidenciales dentro del período analizado, cuando la amenaza de la oposición era mucho menor -alrededor de 26 y 34 puntos porcentuales de margen en las victoriasprobablemente por estar aún muy identificada con la dictadura. Sin embargo, los resultados electorales de 1999 fueron sorpresivamente reñidos con alrededor de tres puntos porcentuales de diferencia entre el candidato oficialista y el opositor, como resultado, por primera vez fue necesario organizar una segunda vuelta de votaciones con las primeras dos mayorías. Por lo tanto, desde ese momento los gobiernos de la Concertación tuvieron un mayor nivel de preocupación como en las elecciones del 2005 (siete puntos porcentuales de margen de victoria), y mucho más aún en el 2009 (tres puntos de margen negativo) cuando perdieron las elecciones presidenciales (Tabla 1). Aquel nivel de competitividad podría verse reflejado en la cantidad de recursos destinados a beneficios habitacionales en esas dos elecciones. 


\section{FIGURA 2. CRECIMIENTO ANUAL DEL PRESUPUESTO.}

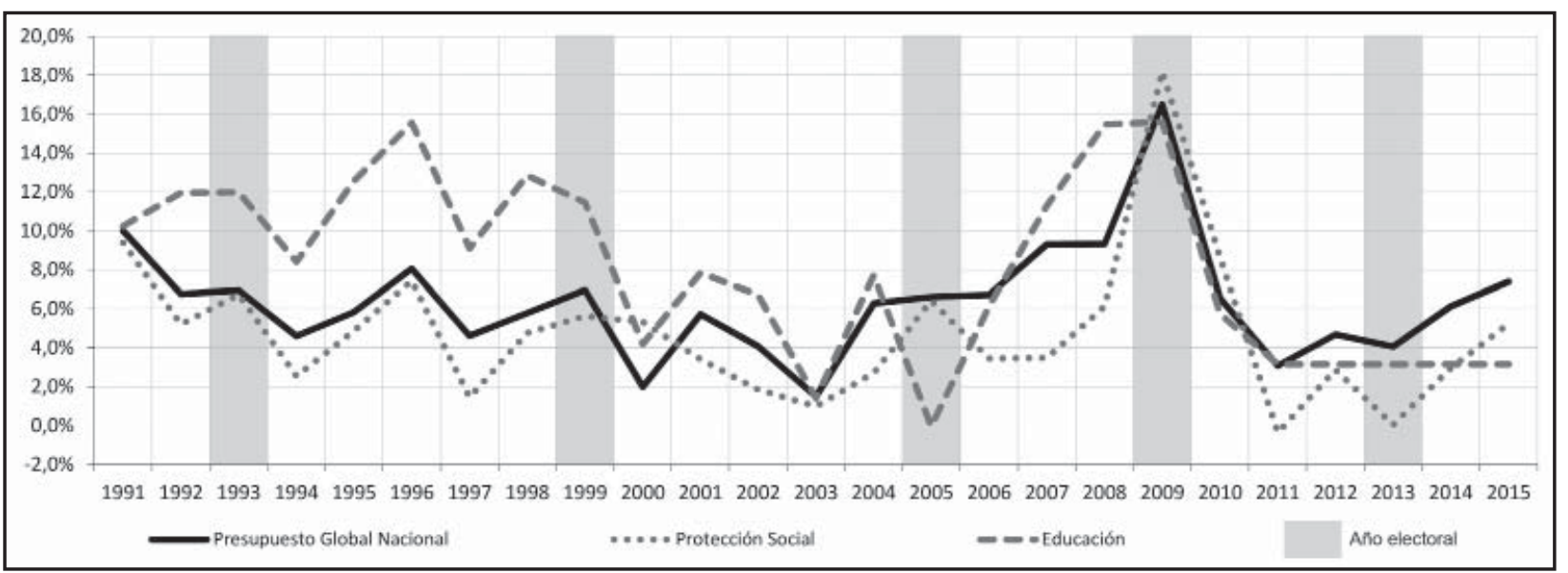

Fuente: Elaboración propia en base a datos de Chile Ministerio de Hacienda, s.f.

A la luz del comportamiento presupuestario a contar de 1990, la pregunta es si los gobiernos manejaron los presupuestos de asistencia social y específicamente el gasto relativo a vivienda para dar una señal de eficiencia o como acción de populismo para año electoral. Primero, considerando las condiciones expuestas por la teoría, los juegos de señales requieren de la existencia de información asimétrica; incentivos para mentir; una acción de señalización costosa; y una acción dependiente de la información oculta. El manejo del presupuesto público en años electorales en Chile cumple con todas las condiciones. A pesar de que Chile ha sido considerado un ejemplo de democracia y progreso económico en Latinoamérica, aún es un país en desarrollo, lo que implica una porción importante de población rural, bajos niveles de educación, falta de independencia de los medios de comunicación y, hasta la década pasada con una baja penetración de tecnologías de la información. Más aún, si se considera la complejidad de los datos presupuestarios, existe un contexto evidente para que se produzca asimetría de información. Dado que la continuidad del gobierno depende de las elecciones presidenciales, la coalición gobernante naturalmente pretende permanecer en el poder $y$, como los votantes deciden quien está en el gobierno basándose en el comportamiento de los políticos, las 
autoridades tienen un incentivo para mentir con el fin de hacer creíble sus capacidades.

A pesar de que en Chile no existe la posibilidad de reelección inmediata de presidente, la dinámica es básicamente la misma debido a que el escenario político estuvo configurado por dos grandes coaliciones que abarcaron prácticamente todo el espectro político. La acción de manejar el presupuesto de asistencia social es claramente costosa y requiere de esfuerzo, dado que el gobierno tiene que redistribuir recursos desde otros sectores que podrían ser conflictivos en el largo plazo tales como educación y salud. Además, como la información oculta es la capacidad del gobierno de garantizar la provisión de bienes y servicios, el aumento del gasto en asistencia social depende de la capacidad de la autoridad para lograrlo. En resumen, cuando la autoridad asigna más recursos en vivienda como una señal, está cambiando las creencias del votante acerca de la rentabilidad del gobierno; si este gobierno es capaz de gastar esa cantidad de recursos, también podría hacer lo mismo para garantizar nuestro bien social común, por tanto garantizaría rentabilidad social. En este caso el manejo del presupuesto resulta ser una señal de eficiencia como gobernantes.

Por otra parte, el manejo del presupuesto para años electorales podría ser considerado como una maniobra populista. Según Canes-Wrone et al., (2001) y Maskin y Tirole (2004), el populismo sucede cuando hay información asimétrica, posibilidad de la autoridad de tomar acciones unilaterales, incentivos electorales, y posibilidad de selección negativa. Como fue mencionado antes, la información entre oficialistas y votantes en Chile es asimétrica. También fue explicado que la autoridad tiene el poder de elaborar el presupuesto con sus glosas y dado las restricciones del parlamento siempre sería aprobado, de esta forma, el presidente es capaz de tomar acciones unilaterales. Las dos coaliciones se comportan tal como si fueran candidatos individuales, por lo tanto, existen incentivos electorales dado que ambos desean que el próximo presidente pertenezca a las filas de su coalición. Finalmente, la principal condición que implica la presencia de populismo es cuando la autoridad ejecuta acciones que no reflejan sus creencias acerca de las políticas apropiadas para ese momento en función del bien común. En este sentido, es posible inferir que el aumento en el crecimiento del presupuesto para vivienda en los años 2005 y 2009 respondió más bien a reacciones demagógicas ante la alta probabilidad de que los candidatos opositores en aquel entonces ganaran la elección, mientras que en los noventa la autoridad no se vio en la necesidad de manejar severamente el presupuesto público en años electorales, dado que la amenaza de la oposición no era significativa.

Prueba de que el aumento de la inversión en vivienda podría haber sido más bien un acto populista 
es que mientras las necesidades de unidades habitacionales fueron disminuyendo rápidamente, la inversión en este ítem se incrementó en los años electorales cuando el déficit era el menor del periodo analizado. Los niveles del déficit habitacional fueron decreciendo sostenidamente desde 1990 con un leve estancamiento y alza en los últimos años lo que no opacó el gran avance alcanzado durante veinte años de gobiernos de la Concertación logrando una disminución de prácticamente la mitad del déficit de 1990 transitando de la necesidad de 942.000 unidades en ese año a 421.000 el año 2009, según la encuesta CASEN. Como debiera suponerse, la inversión tendría que haber disminuido, considerando que el problema era mucho menos dramático en comparación a los de otras carteras tales como salud y educación. Sin embargo, como se puede observar en la Figura 4, el nivel de inversión en vivienda tuvo un aumento explosivo en el año electoral del 2009 y un importante crecimiento respecto del año anterior (Figura 3).

Cabe mencionar que el comportamiento del gobierno en el año 2009 también se debió a una medida de orden macroeconómico para hacer frente a la crisis económica de aquel año, donde la construcción masiva de vivienda con mano de obra intensiva parecía una buena idea para evitar el alza de los niveles de cesantía dada la disminución de inversión privada en el sector construcción, el que además emplea a decenas de miles de personas sin calificación para el mundo laboral, situación que por lo tanto los transformaría en los primeros afectados de la crisis económica. Si bien es complejo aislar esta situación de las intenciones electorales de cada gobierno, al menos es posible establecer la evidencia señalada para comprender el comportamiento del oficialismo desde un punto de vista histórico.

\section{Reflexiones sobre el manejo de presupuesto en vivienda}

Si bien el manejo presupuestario habitacional motivado por la competencia política no puede explicarse únicamente desde una perspectiva cuantitativa, es útil comprenderlo para eventualmente establecer nuevas variables que inciden en la inversión para vivienda. Al comparar la variación de los factores analizados siguiendo los modelos del juego de señales y del populismo es posible establecer las eventuales motivaciones detrás de las decisiones políticas para definir la inversión en la vivienda en Chile.

La definición y distribución del presupuesto público para años electorales en Chile, lejos de ser una asignación neutral de recursos, entrega signos de que ha correspondido más bien al manejo del presupuesto desde 1990 según el estudio realizado. La glosa de asistencia social se ha incrementado continuamente y con mayor intensidad que 


\section{FIGURA 3. CRECIMIENTO DEL PRESUPUESTO TOTAL E INVERSIÓN EN VIVIENDA.}

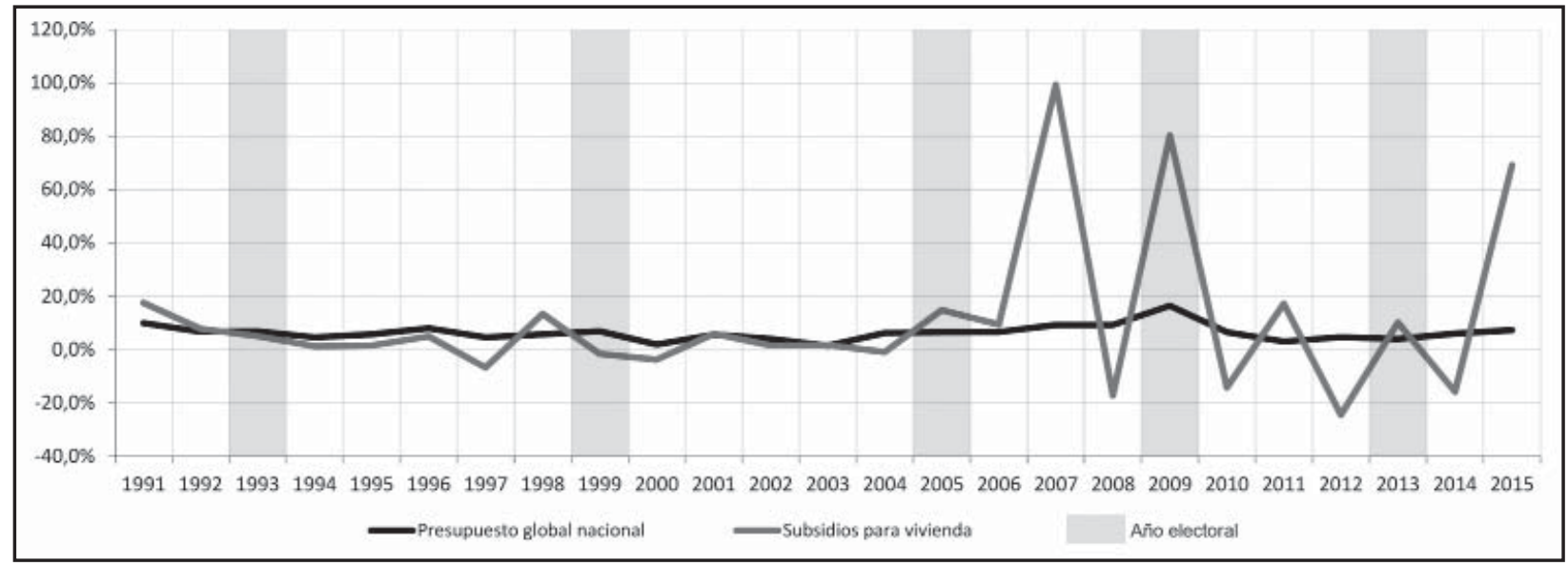

Fuente: Elaboración propia en base a datos del Observatorio Habitacional y Chile Ministerio de Hacienda, s.f..

\section{FIGURA 4. EVOLUCIÓN DE INVERSIÓN EN VIVIENDA Y EL DÉFICIT HABITACIONAL.}

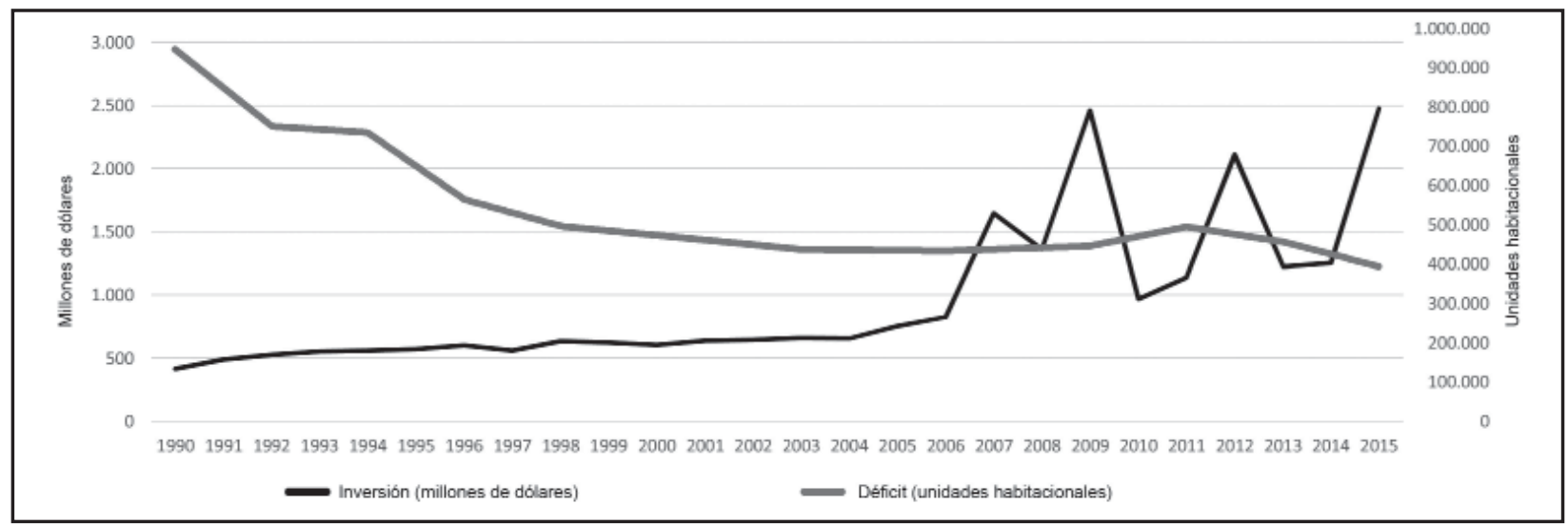

Fuente: Elaboración propia en base a datos del Observatorio Habitacional y el Ministerio de Vivienda y Urbanismo. 
el presupuesto global justo antes de las elecciones presidenciales, lo que podría ser interpretado como una señal de la eficiencia de los gobiernos. Dado que los gobiernos, en efecto, ejecutan gastos para responder a las necesidades reales de las personas, cabría la posibilidad de que las autoridades postergaron las inversiones en obras y programas más visibles durante sus gobiernos para ejecutarlas en años electorales. Sin embargo, mientras la distribución del presupuesto ha sido efectiva para solucionar problemas sociales quizás no es óptimo que lo haga de esta forma.

Asumiendo que el gasto público en Chile no ha correspondido a una asignación inadecuada de recursos, la acción podría ser catalogada como una señal de eficiencia, pero el alto manejo presupuestario, seguido por un margen estrecho de victoria en las elecciones presidenciales del 2005 y 2009 y el bajo déficit histórico de vivienda, pone en duda esa interpretación. Esto sugeriría que, en la práctica, el gobierno no ha estado necesariamente buscando las políticas más adecuadas dadas las necesidades contingentes en el país, por lo tanto, la señal de eficiencia se transformaría en un acto de populismo en aquellas elecciones. Este problema también puede ser explicado por la existencia de un juego repetitivo, que incluye a la reputación como una variable. Así, mientras el gobierno manejó el presupuesto, los votantes podrían haber comenzado a percatarse de la asignación tendenciosa de recursos, hasta que el electorado se inclinó por elegir a la oposición para un nuevo gobierno en el año 2010. Sobre todo, los electores habrían tenido la capacidad de reconocer algún grado de populismo en los políticos gracias a las mejoras de educación de las últimas décadas, el acceso a tecnologías de la información y a la transparencia del gobierno. Tal como argumentan Brender y Drazen (2005), los CPP disminuyen a lo largo del tiempo junto con el aprendizaje de los países sobre los procesos electorales competitivos, lo que, resumiendo, implica más y mejor democracia.

En síntesis, el estudio conduce a la construcción de una hipótesis sobre la correlación entre asignaciones de presupuesto en vivienda y la competitividad electoral, situación que claramente requiere de más estudios y mayores antecedentes para verificar la eventual causalidad, que permitiría inferir y distinguir la distribución del presupuesto en vivienda como actos para evidenciar capacidad o populismo en periodos electorales.

\section{Referencias bibliográficas}

Aidt, T.S., Veiga, F., y Veiga, L. (2011). Election results and opportunistic policies: A new test of the rational political business cycle model. Public choice, 148(1-2), 21-44. https://doi.org/10.1007/ s11127-010-9644-3 
Alt, J.E. y Lassen, D.D. (2006). Transparency, political polarization, and political budget cycles in OECD countries. American Journal of Political Science, 50(3), 530-550. https://doi. org/10.1111/j.1540-5907.2006.00200.x

Banco Mundial. (s.f.). Chile. Recuperado de http://data. worldbank.org/country/chile

Brender, A. y Drazen. (2005). Political budget cycles in new versus established democracies. Journal of Monetary Economics, 52(7), 1271-1295. https://doi. org/10.1016/j.jmoneco.2005.04.004

Canes-Wrone, B., Herron, M.C., y Shotts, K.W. (2001). Leadership and pandering: A theory of executive policymaking. American Journal of Political Science, 45(3), 532-550. https://doi.org/10.2307/2669237

Castro, V. y Martins, R. (2016). Are there political cycles hidden inside government expenditures? Applied Economics Letters, 23(1), 34-37. https://doi.org/10. 1080/13504851.2015.1047084

Chile Ministerio de Hacienda. (s.f.) Estado de operaciones del gobierno central 1990 - 2017. Recuperado de http://www.dipres.gob.cl/594/w3-propertyvalue-15494.html

Chile Ministerio de Planificación y Cooperación. (1998). Impacto de la política habitacional chilena. Santiago: Autor.

Chile Ministerio de Vivienda y Urbanismo. (2004). Un siglo de políticas en vivienda y barrio. Santiago: Autor.

Drazen, A. y Eslava, M. (2010). Electoral manipulation via voter-friendly spending: Theory and evidence.
Journal of Development Economics, 92(1), 39-52. https://doi.org/10.1016/j.jdeveco.2009.01.001

Efthyvoulou, G. (2012). Political budget cycles in the European Union and the impact of political pressures. Public Choice, 153(3-4), 295-327. https://doi. org/10.1007/s11127-011-9795-x

Eibl, F. y Lynge-Mangueira, H. (2017). Electoral confidence and political budget cycles in non-OECD countries. Studies in Comparative International Development, 52(1), 1-19. https://doi.org/10.1007/ s12116-016-9230-x

Katsimi, M. y Sarantides, V. (2012). Do elections affect the composition of fiscal policy in developed, established democracies? Public Choice, 151(1), p. 325362. https://doi.org/10.1007/s11127-010-9749-8

Khemani, S. (2004). Political cycles in a developing economy: effect of elections in the Indian states. Journal of Development Economics, 73(1), 125-154. https://doi.org/10.1016/j.jdeveco.2003.01.002

Maskin, E. y Tirole, J. (2004). The politician and the judge: Accountability in government. American Economic Review, 94(4), 1034-1054. https://doi. org/10.1257/0002828042002606

Nordhaus, W.D. (1975). The political business cycle. The Review of Economic Studies, 42(2), 169-190. https:// doi.org/10.2307/2296528

Persson, T. y Tabellini, G. (1990). Macroeconomic policy, credibility and politics. Abingdon: Taylor \& Francis. 
Persson, T. y Tabellini, G. (2003). Do electoral cycles differ across political systems? Milan: IGIER.

Rogoff, K. (1987). Equilibrium political budget cycles. Cambridge, MA.: National Bureau of Economic Research.

Rogoff, K. y Sibert, A. (1988). Elections and macroeconomic policy cycles. The Review of Economic Studies, 55(1), 1-16. https://doi.org/10.2307/2297526

Shi, M. y Svensson, J. (2002). Conditional political budget cycles. London: Centre for Economic Policy Research.

Shi, M. y Svensson, J. (2006). Political budget cycles: Do they differ across countries and why? Journal of Public Economics, 90(8-9), 1367-1389. https:// doi.org/10.1016/j.jpubeco.2005.09.009

Spence, M. (1973). Job market signaling. The Quarterly Journal of Economics, 87(3), p. 355-374. https://doi. org/10.2307/1882010

Vergne, C. (2009). Democracy, elections and allocation of public expenditures in developing countries. European Journal of Political Economy, 25(1), 63-77. https://doi.org/10.1016/j.ejpoleco.2008.09.003 